\title{
دور الكاينيتين والسماد المركب NPKZn في فعالية بعض مضادات الاكسدة لنبات القلقل الحلو Capsicum annuum L.
}

The Kinetin Role and Compound Fertilizer NPKZn in the Effectiveness of some Antioxidants to Sweet Pepper Capsicum annuum L. subjected to Salt Stress

\author{
سعاد عبد سيد* \\ كلية التربيةً/ ابن الهيثم/ جامعة بغداد

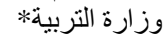 \\ A.J.H. Al-Saedi \\ S.A.S. Al-jalaly* \\ College of Education / Ibn Al-Hatham / Baghdad University \\ * Ministry of Education
}

E-mail: suadaljalali@Yahoo.com

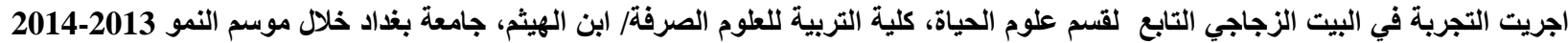

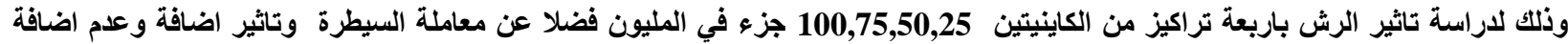

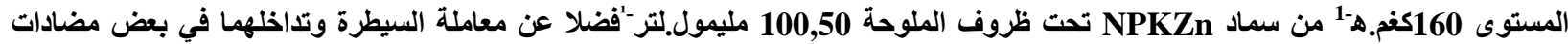

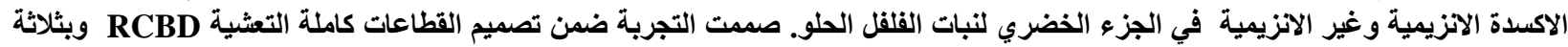

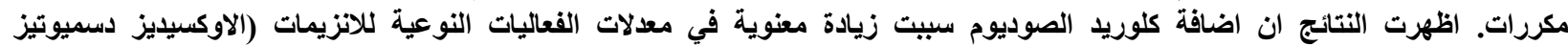

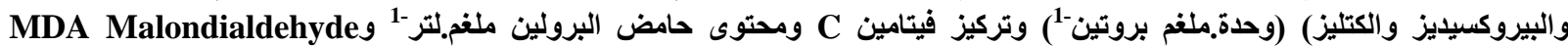

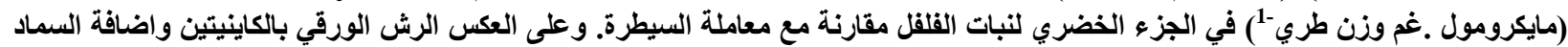

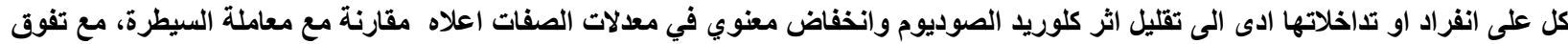

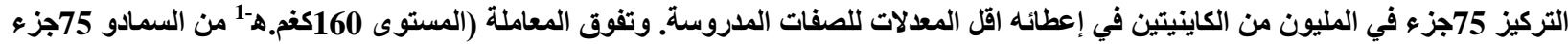
في المليون من الكاينيتين) في إعطائه افضل المئن المعدلات للصفات اعلاته اعلاه.

الكلمات المفتاحية: الكاينيتين، سماد NPKZn، مضادات اكسدة

Abstract

The experiment was carried out in the green house of Biology Department, College of Education for Pure Science/ Ibn AL-Haitham, Baghdad University, during the growing season of 2013- 2014, to study the influence of foliar application of four concentrations of kinetine $25,50,75,100 \mathrm{ppm}$ in addition to the control treatment, appling and non appling of $160 \mathrm{Kg}^{-1} \mathrm{H}^{-1} \mathrm{NPKZn}$ fertilizer, and two concentrations of sodium chloride $50,100 \mathrm{mM} . \mathrm{L}^{-1}$ instead of control treatment, and their interactions on Some Antioxidant Enzyme and non-Enzyme of vegetative part of Capscum annum L. The experiment was designed by using Randomized Complete Block Design (RCBD) with three replications. Results indicated that application of sodium chloride caused a significant increase in the average enzyme activities (Superoxide dismutase, Peroxidase, Catalase) (unite mg protein ${ }^{-1}$ ), vitamin $C$ concentration, proline aced content $\left(\mathrm{mg}^{-\mathrm{L}^{-1}}\right)$ and Malondialialdehyde content ( $\mu$ M.gm F.W. ${ }^{-1}$ ) compared with the control, on the contrary the spray of the kinetine and aplication of the fertilizer both individually or its interventions led to decrease the injury of sodium chloride and a significant reduction in rates of details mentioned above effect of. The surpass of $75 \mathrm{ppm}$ of the kinetine which agave the less mean of characteristics studied and superiority of the treatment (level $160 \mathrm{Kg}^{-1} \mathrm{H}^{-1} \mathrm{NPKZn}$ fertilizer and $75 \mathrm{ppm}$ of kinetine in giving the best averages of characteristics above.

Key words: kinetine, NPKZn fertilizer, antioxidants

المقدمة

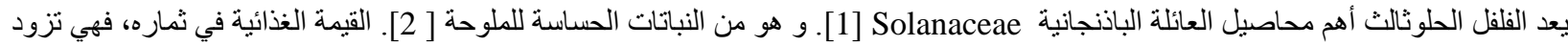

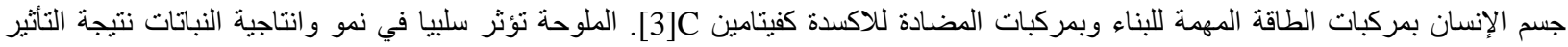

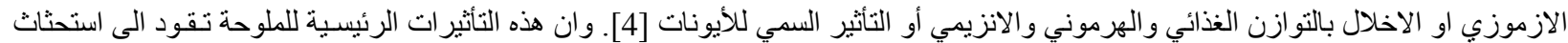

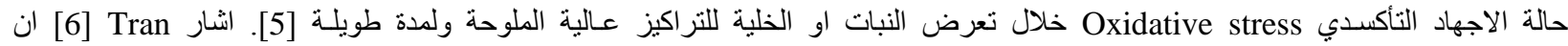

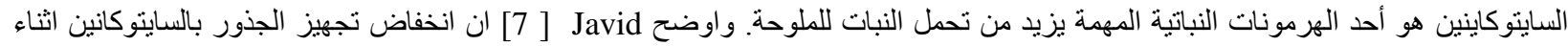

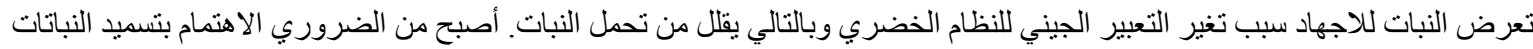




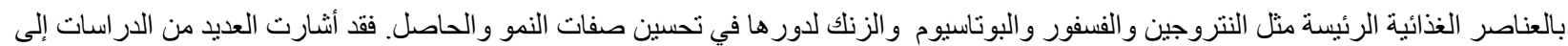

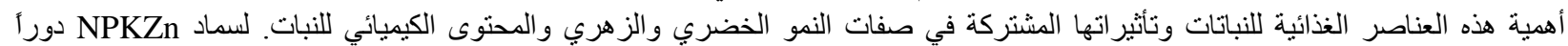

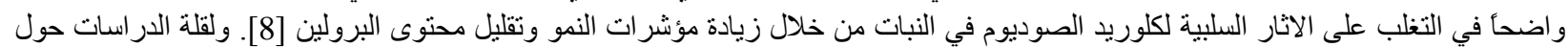

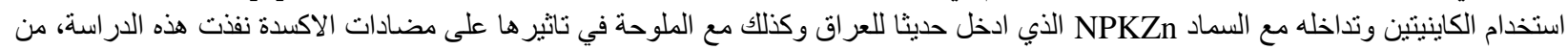

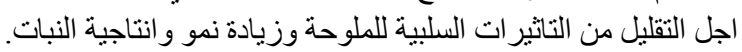

المواد وطرق العمل من التير

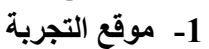

اجريت التجربة في البيت الزجاجي التنابع لقنم علوم الحياة، كلية التربية للعلوم الصرفة ـ ابن الهيثم، جامعة بغداد خلال موسم النمو 2013-2014 باستعمال الاصص البلاستيكية سعة 8 كغ تربة التربة

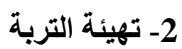

جلبت التربة من احد الحقول الزراعية التابع للهيئة العامة للبحوث الزر اعية في منطقة أبو غريب على عمق (-0-30) سم وتم تجفيفها وطحنها ونخلها

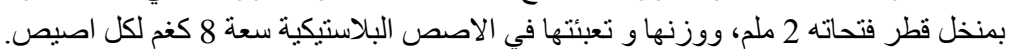
3- ت تسميد التربة

سمدت التربة الموجودة في الأصص قبل عملية زراعة الثتلات بسماد NPKZn تركي المنثـأ بمقار 0.65 غم لكل اصيص على اساس 160 كغم لالهكتار ، اضافة للمعاملة صفر. 4ـ تصميم التجربة وزراعة الثتلات صمدت التجربة على وفق تصميم القطاعات كاملة التعشية بوصفيها تجربة عاملية (2×5 ×3 ×3 ) وبثلاثة مكررات وبذللك تضمنت التجربة (90) اصيصا، تمت عملية زر اعة الثتلات بتاريخ 2014/2/4 اذ زرعت 3 شتلاتلات لكل اصيص. 5 الري الري

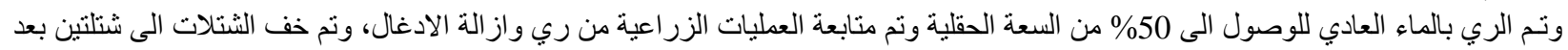

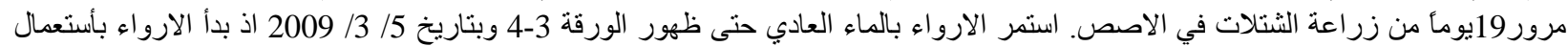

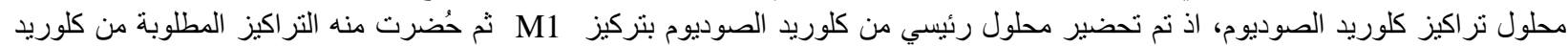

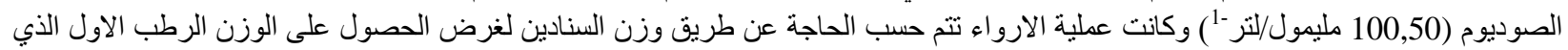
بدأت فيه التجربة.

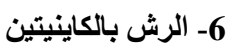

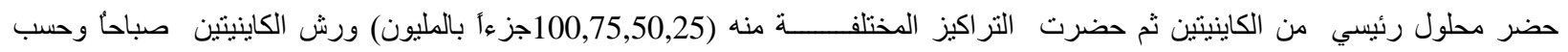

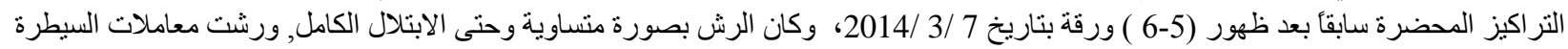

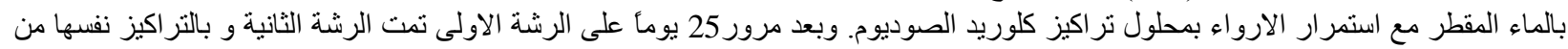

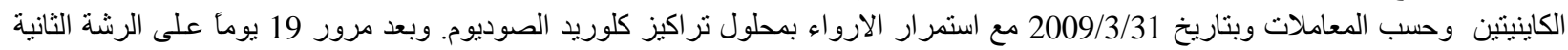

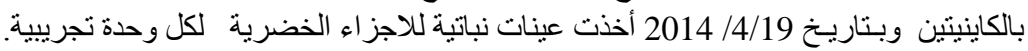
الصفات المدروسة

1 - 1 تقير فعالية الإنزيمات المضادة للأكسدة

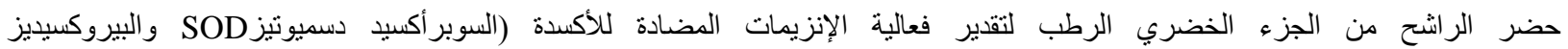

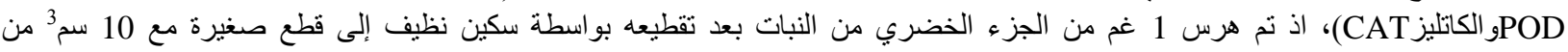

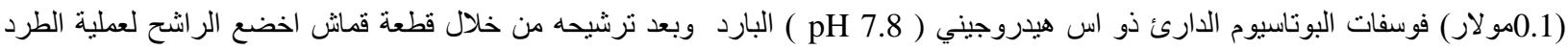

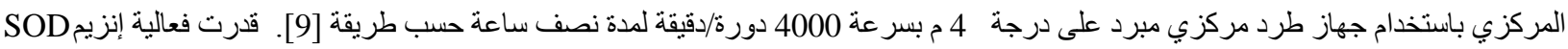

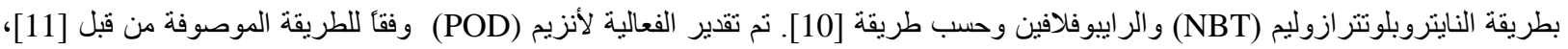

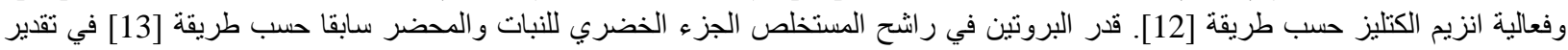

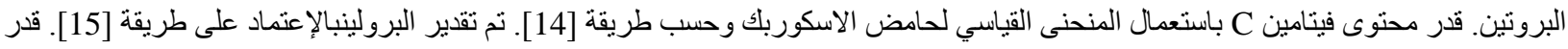

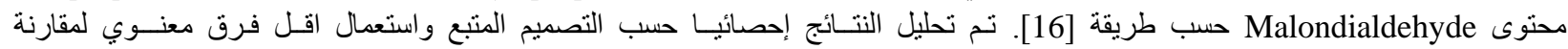
الكنوسطات الحسايية للمعاملات عند مستوى احتمال 0.05 [17].

النتائج والمناقشة

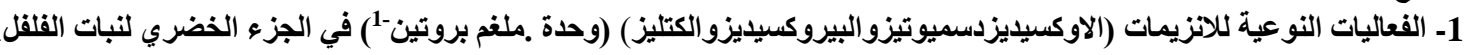

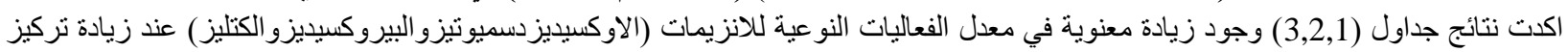

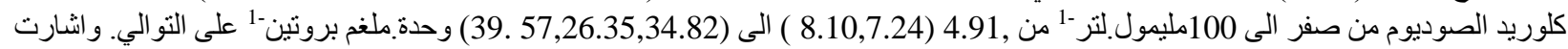

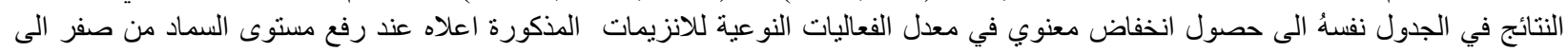

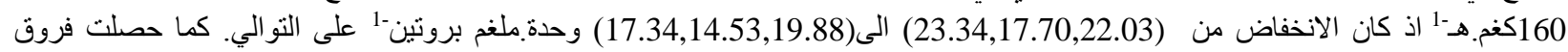

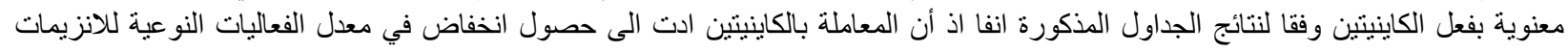

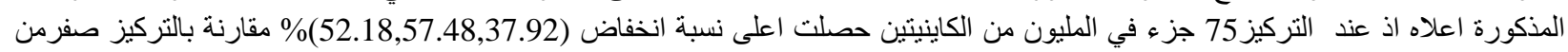

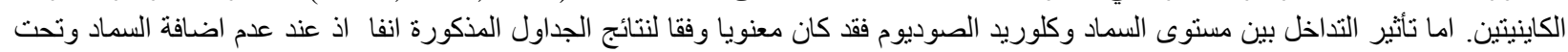

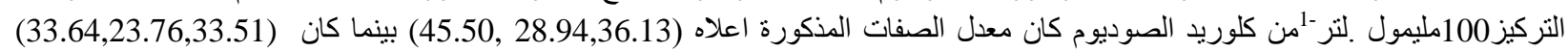

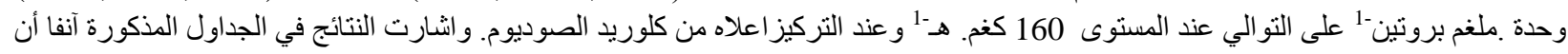




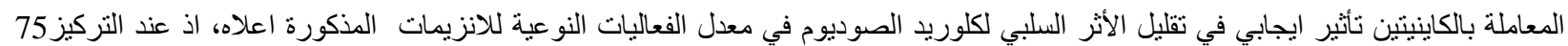

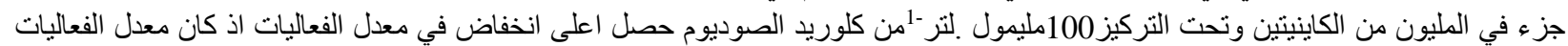

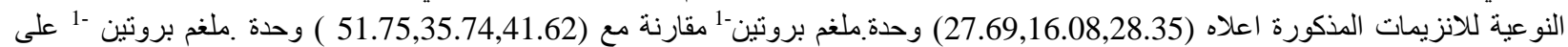

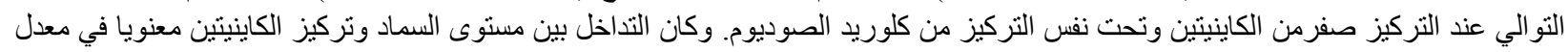

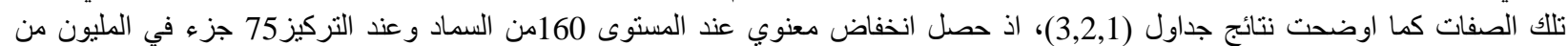

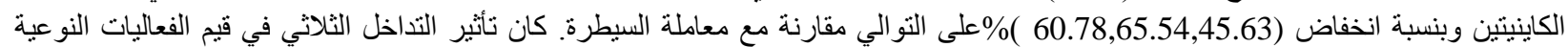

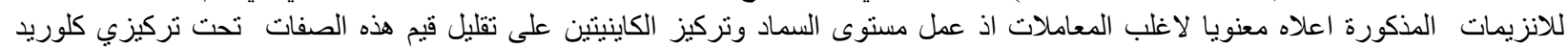

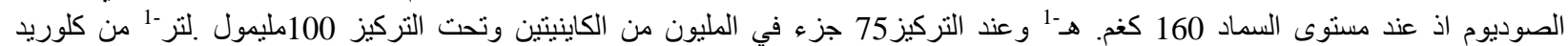

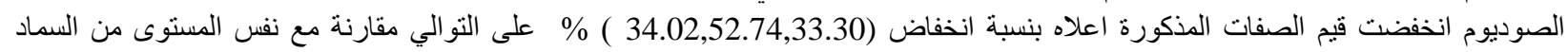

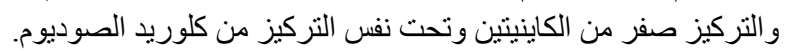

جدول (1): تاثير الرش بالكاينيتين واضافة سماد NPKZn في الفعالية النوعيةلانزيمSOD (وحدة .ملفم بروتين -1) في الجزء الخضري لنبات الفلقل

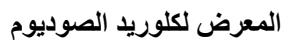

\begin{tabular}{|c|c|c|c|c|c|c|c|}
\hline \multirow{2}{*}{ متوسطتاثير تداخل كلوريد الصوديوم } & \multicolumn{5}{|c|}{ تراكيز الكاينيتين ( جزء في المليون) } & \multirow{2}{*}{ 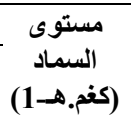 } & \multirow[b]{2}{*}{ تركيز كلوريد الصوديوم (مليمول.لتر-1) } \\
\hline & 100 & 75 & 50 & 25 & $\mathbf{0}$ & & \\
\hline 8.34 & 8.23 & 4.32 & 7.45 & 8.80 & 12.92 & 0 & \multirow{2}{*}{$\mathbf{0}$} \\
\hline 6.13 & 8.01 & 3.35 & 4.09 & 6.87 & 8.32 & 160 & \\
\hline 21.62 & 22.18 & 17.41 & 20.06 & 22.47 & 25.96 & $\mathbf{0}$ & \multirow{2}{*}{50} \\
\hline 20.00 & 21.16 & 14.29 & 18.82 & 21.39 & 24.32 & 160 & \\
\hline 36.13 & 37.16 & 29.84 & 32.91 & 37.78 & 42.96 & $\mathbf{0}$ & \multirow{2}{*}{100} \\
\hline \multirow[t]{2}{*}{33.51} & 34.70 & 26.86 & 30.63 & 35.07 & 40.27 & 160 & \\
\hline & 21.91 & 16.01 & 18.99 & 22.06 & 25.79 & \multicolumn{2}{|r|}{ متوسط تاثير تركيز الكاينيتين } \\
\hline 1.293 & $\begin{array}{l}1 . \\
2.8\end{array}$ & 80 & \multicolumn{3}{|c|}{ معدل تاثير الكاينيتين } & \multicolumn{2}{|r|}{ LSD (0.05) } \\
\hline \multicolumn{8}{|c|}{ متوسط تأثيرتداخل كلوريد الصوديوم" × تركيز الكاينتين } \\
\hline مته سط تأثر كله ر بـ الصه ديه م & \multicolumn{5}{|c|}{ تركيز الكاينتين } & \multirow{2}{*}{\multicolumn{2}{|c|}{ كلوريد الصوديوم }} \\
\hline مسوسط لابيرلوريد الصوديوم & 100 & 75 & $\mathbf{5 0}$ & 25 & $\mathbf{0}$ & & \\
\hline 7.24 & 8.12 & 3.84 & 5.77 & 7.84 & 10.62 & & $\mathbf{0}$ \\
\hline 20.81 & 21.67 & 15.85 & 19.44 & 21.93 & 25.14 & & 50 \\
\hline 34.82 & 35.93 & 28.35 & 31.77 & 36.43 & 41.62 & & 100 \\
\hline \multirow[t]{2}{*}{0.914} & \multicolumn{5}{|c|}{$\mathbf{2 . 0 4 4}$} & \multirow{2}{*}{\multicolumn{2}{|c|}{ LSD (0.05) }} \\
\hline & & بنتين & 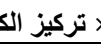 & 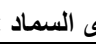 & 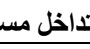 & & \\
\hline \multirow{2}{*}{ متوسط تأثير السماد } & \multicolumn{5}{|c|}{ تركيز الكاينتين } & \multirow{2}{*}{\multicolumn{2}{|c|}{ 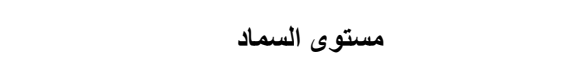 }} \\
\hline & 150 & 100 & 50 & 25 & & & \\
\hline 22.03 & 22.52 & 17.19 & 20.14 & 23.02 & 27.28 & & صفز \\
\hline 19.88 & 21.29 & 14.83 & 17.85 & 21.11 & 24.30 & & \\
\hline 0.746 & \multicolumn{5}{|c|}{1.669} & \multicolumn{2}{|r|}{ LSD $(0.05)$} \\
\hline
\end{tabular}




\begin{tabular}{|c|c|c|c|c|c|c|c|}
\hline \multirow{2}{*}{ متوسط تاثير تاخئ الجزء الخضري } & & & & \multicolumn{4}{|c|}{ لنبات الفلقل المعرض لكلوريد الصوديوم } \\
\hline & \multicolumn{5}{|c|}{ تراكيز الكاينيتين ( جزء في المليون) } & \multirow{2}{*}{ 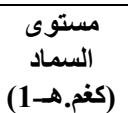 } & \multirow{2}{*}{ 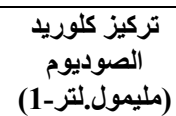 } \\
\hline الصوديوم × السماد مستوى & 100 & 75 & 50 & 25 & $\mathbf{0}$ & & \\
\hline 5.27 & 5.76 & 2.77 & 4.03 & 5.84 & 7.96 & 0 & \multirow[t]{2}{*}{ ) } \\
\hline 4.55 & 5.45 & 2.45 & 2.99 & 5.85 & 6.00 & 160 & \\
\hline 19.24 & 21.13 & 10.35 & 14.17 & 22.69 & 27.85 & $\mathbf{0}$ & \multirow{2}{*}{50} \\
\hline 13.85 & 12.68 & 9.05 & 12.33 & 13.22 & 21.95 & 160 & \\
\hline 45.50 & $\mathbf{5 0 . 5 0}$ & 28.32 & 33.70 & 52.50 & 62.50 & $\mathbf{0}$ & \multirow[b]{2}{*}{100} \\
\hline \multirow[t]{2}{*}{33.64} & 34.85 & 27.05 & 30.14 & 35.15 & 41.00 & 160 & \\
\hline & 21.73 & 13.33 & 16.23 & 22.54 & 27.88 & \multicolumn{2}{|c|}{ متوسط تاثير تركيز الكاينيتين } \\
\hline \multirow[t]{2}{*}{1.086} & \multicolumn{2}{|c|}{$\begin{array}{l}0.991 \\
2.428\end{array}$} & \multicolumn{3}{|c|}{ 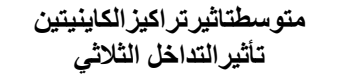 } & \multicolumn{2}{|c|}{ LSD $(0.05)$} \\
\hline & \multicolumn{5}{|c|}{ متوسط تأثيرتداخل كلوريد الصوديوم × تركيز الكاينتين } & \multirow{2}{*}{\multicolumn{2}{|c|}{ تركيز كلوريد الصوديوم }} \\
\hline متوسط تأثيركلوريد الصوديوم & 100 & 75 & 50 & 25 & 0 & & \\
\hline 4.91 & 5.61 & 2.61 & 3.51 & 5.85 & 6.98 & \multicolumn{2}{|r|}{ 0 } \\
\hline 16.54 & 16.91 & 9.70 & 13.25 & 17.96 & 24.90 & \multicolumn{2}{|c|}{50} \\
\hline 39.57 & 42.68 & 27.69 & 31.92 & 43.83 & 51.75 & \multicolumn{2}{|c|}{100} \\
\hline \multirow[t]{2}{*}{0.768} & \multicolumn{5}{|c|}{1.717} & \multirow{2}{*}{\multicolumn{2}{|c|}{ LSD (0.05) }} \\
\hline & \multicolumn{5}{|c|}{ متوسط تاثير تداخل مستوى السماد × تركيز الكاينيتين } & & \\
\hline متوسط تأثير السماد & 100 & 75 & 50 & 25 & 0 & \multicolumn{2}{|c|}{ 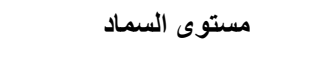 } \\
\hline 23.34 & 25.80 & 13.81 & 17.30 & 27.01 & 32.77 & \multicolumn{2}{|c|}{ صفر } \\
\hline 17.34 & 17.66 & 12.85 & 15.15 & 18.07 & 22.98 & \multicolumn{2}{|c|}{160} \\
\hline 0.627 & \multicolumn{5}{|c|}{1.402} & \multicolumn{2}{|c|}{ LSD (0.05) } \\
\hline
\end{tabular}

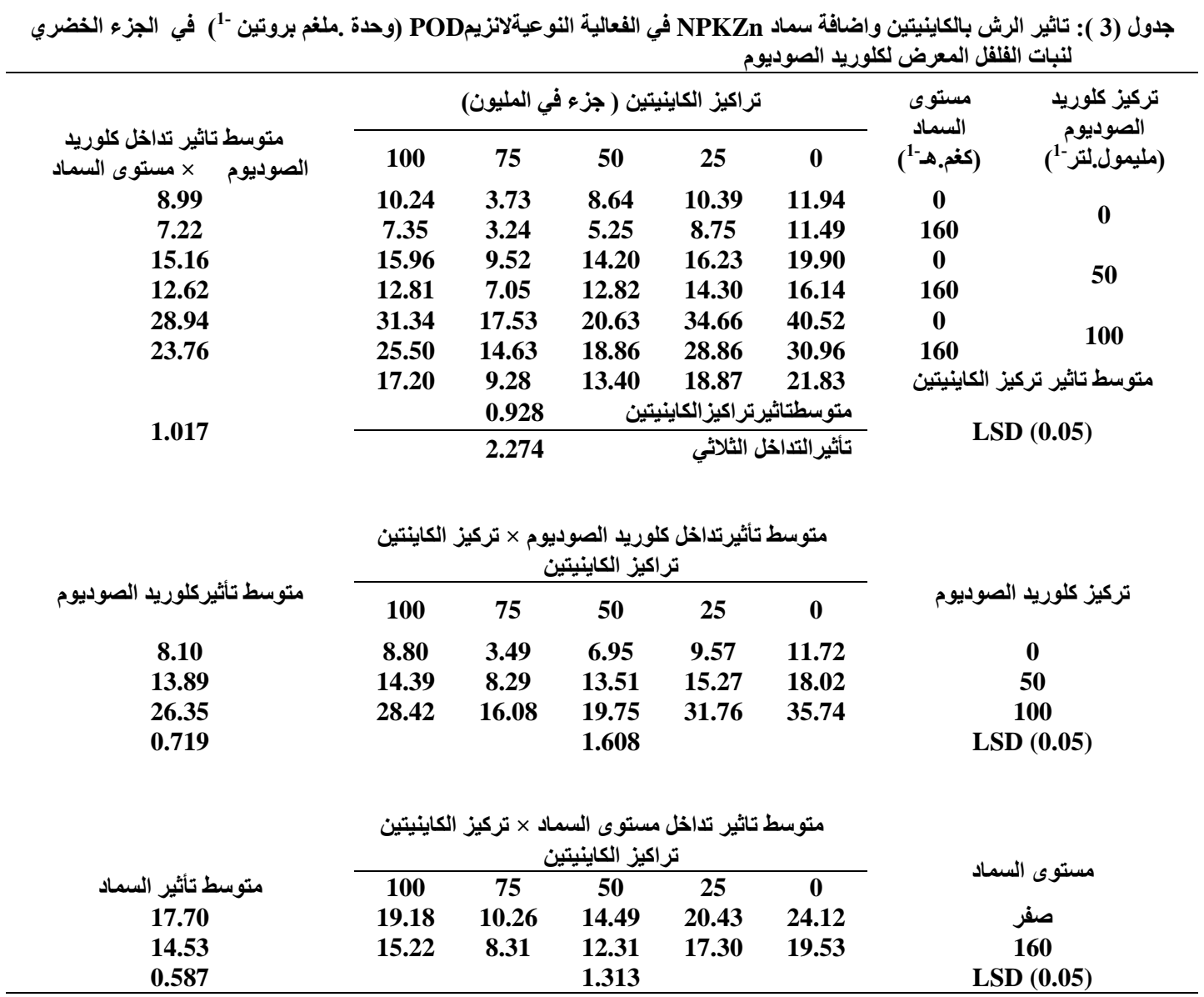


2-تركيز فيتامين CC ومحتوى البرولين (ملغ.لتر-1) وMDA) Malondialdehyde (مايكرومول .غم وزن طري-1) في الجزء الخضري لنبات الفلفل

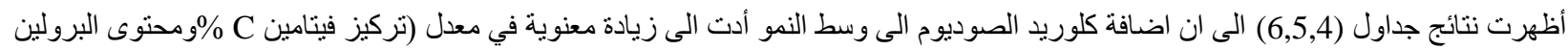

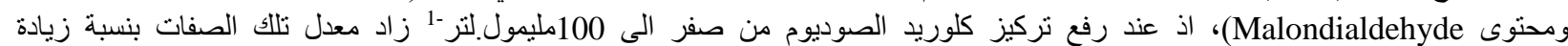

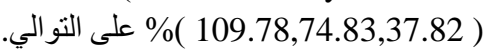

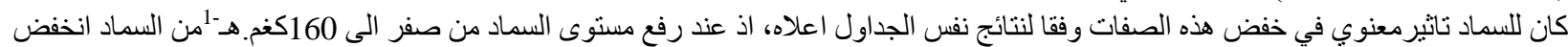

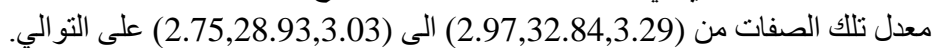

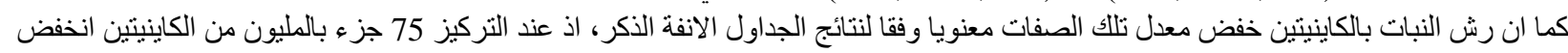

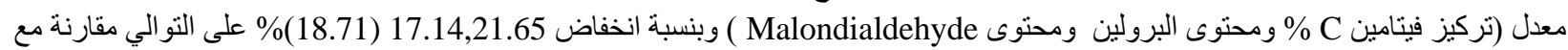
التركيز صفر من الكاينيتين.

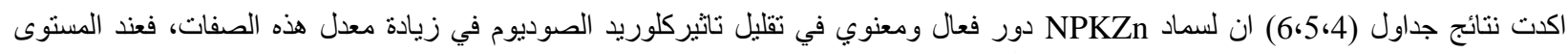

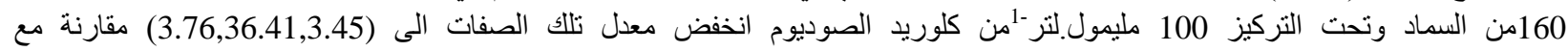

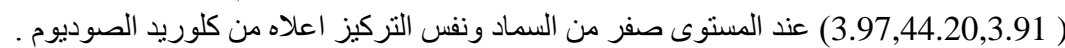

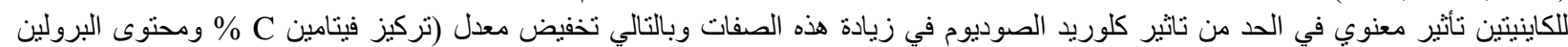

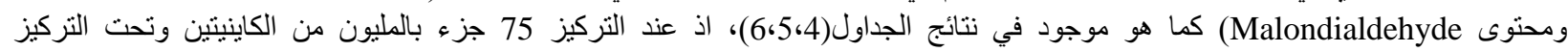

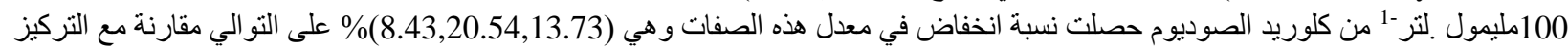

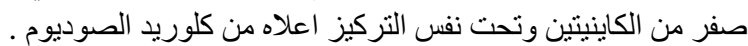

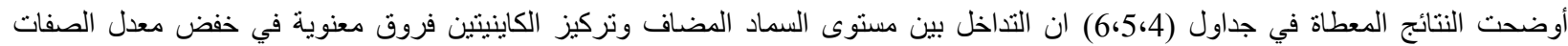

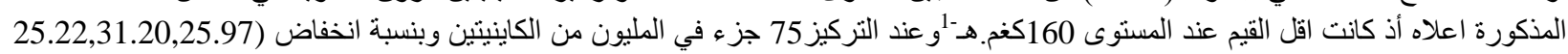
) \% على التو اليمقارنة مع معاملة السيطرة.

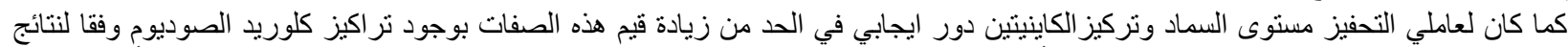

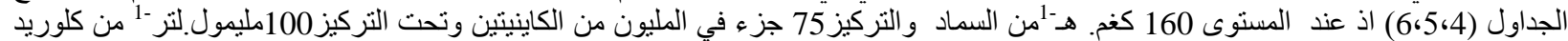

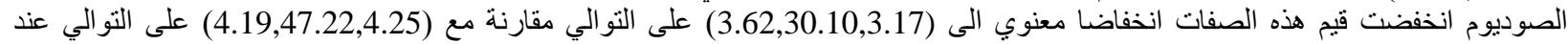
المستوى صفر من السماد و التزكيزصفرمنالكاينيتين ونفس التركيز من كلوريد الصودئ التيوم العلاه، وبذلك كان دور السماد المضاف والرش بالكاينيتين واضح في تقليل قيم هذه الصفة تحت تر اكيز كلوريد الصوديوم.

جدول ( 4): تاثير الرش بالكينتين واضافة سماد NPKZn في محتوى البرولين (مايكروغرام.غم وزن جاف-1) الجزء الخضري لنبات الفلفل

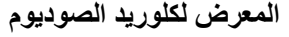

\begin{tabular}{|c|c|c|c|c|c|c|c|}
\hline \multirow{2}{*}{ 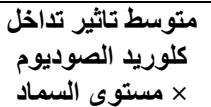 } & \multicolumn{5}{|c|}{ تراكيز الكاينيتين ( جزء في المليون) } & \multirow{2}{*}{ مستوى السماد } & \multirow{2}{*}{ تركيز كلوريا } \\
\hline & 100 & 75 & 50 & & 0 & & \\
\hline 23.79 & 24.30 & 18.52 & 22.56 & 25.45 & 28.11 & 0 & \multirow{2}{*}{$\mathbf{0}$} \\
\hline 22.31 & 23.65 & 17.42 & 20.05 & 24.22 & 26.20 & 160 & \\
\hline 30.53 & 30.60 & 28.21 & 30.30 & 31.13 & 32.43 & 0 & \multirow{2}{*}{50} \\
\hline 28.07 & 27.65 & 26.62 & 27.26 & 28.41 & 30.43 & 160 & \\
\hline 44.20 & 44.05 & 40.20 & 44.01 & 45.50 & 47.22 & $\mathbf{0}$ & \multirow{2}{*}{100} \\
\hline \multirow[t]{2}{*}{36.41} & 39.85 & 30.10 & 30.80 & 40.06 & 41.25 & 160 & \\
\hline & 31.68 & 26.85 & 29.16 & 32.46 & 34.27 & \multicolumn{2}{|c|}{ متوسط تاثير تركيز الكاينيتين } \\
\hline 0.383 & \multicolumn{2}{|c|}{$\begin{array}{l}\mathbf{0 . 3 5 0} \\
\mathbf{0 . 8 5 7}\end{array}$} & \multicolumn{3}{|c|}{ متوسنتاثيرتراكيز الكاينيتين } & \multicolumn{2}{|c|}{ RLSD (0.05) } \\
\hline متوسط تأثيركلوريد & \multicolumn{5}{|c|}{ متوسط تأثيرتداخل كلوريد الصوديوم × تركيز الكاينتين } & \multirow{2}{*}{\multicolumn{2}{|c|}{ تركيز كلوريد الصوديوم }} \\
\hline الصوديوم & 100 & 75 & $\mathbf{5 0}$ & 25 & 0 & & \\
\hline 23.05 & 23.98 & 17.97 & 21.31 & 24.84 & 27.16 & \multicolumn{2}{|c|}{ 0 } \\
\hline 29.30 & 29.13 & 27.42 & 28.78 & 29.77 & 31.43 & \multicolumn{2}{|c|}{50} \\
\hline 40.30 & 41.95 & 35.15 & 37.41 & 42.78 & 44.24 & \multicolumn{2}{|c|}{100} \\
\hline 0.271 & & 0.606 & & & & \multirow{2}{*}{\multicolumn{2}{|c|}{ LSD (0.05) }} \\
\hline \multicolumn{6}{|c|}{ متوسط تاثير تداخل مستوى السماد × تركيز الكاينيتين } & & \\
\hline متوسط تأثير السماد & 100 & 75 & $\mathbf{5 0}$ & 25 & 0 & \multicolumn{2}{|c|}{ مستوى السماد } \\
\hline 32.84 & 32.98 & 28.98 & 32.29 & 34.03 & 35.92 & \multicolumn{2}{|c|}{ صفر } \\
\hline 28.93 & 30.38 & 24.71 & 26.04 & 30.90 & 32.63 & \multirow{2}{*}{\multicolumn{2}{|c|}{$\begin{array}{c}160 \\
\text { LSD }(0.05)\end{array}$}} \\
\hline 0.221 & & 0.495 & & & & & \\
\hline
\end{tabular}




\begin{tabular}{|c|c|c|c|c|c|c|c|}
\hline \multirow[b]{2}{*}{ الصوديوم × مستوى تاثير تداخل كلوريد } & \multicolumn{5}{|c|}{ تراكيز الكاينيتين ( جزء في المليون) } & \multirow{2}{*}{ مستوى السماد } & \multirow{2}{*}{ 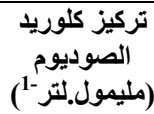 } \\
\hline & 100 & 75 & 50 & 25 & $\mathbf{0}$ & & \\
\hline 2.74 & 2.70 & 2.48 & 2.69 & 2.84 & 3.00 & 0 & \multirow{2}{*}{ ) } \\
\hline 2.59 & 2.69 & 2.05 & 2.59 & 2.71 & 2.91 & 160 & \\
\hline 3.21 & 3.35 & 2.72 & 3.12 & 3.37 & 3.50 & $\mathbf{0}$ & \multirow{2}{*}{50} \\
\hline 3.25 & 3.05 & 2.74 & 3.97 & 3.07 & 3.41 & 160 & \\
\hline 3.45 & 3.54 & 3.17 & 3.33 & 3.76 & 3.46 & $\mathbf{0}$ & \multirow{3}{*}{100} \\
\hline \multirow[t]{2}{*}{3.95} & 4.05 & 3.49 & 3.85 & 4.09 & 4.25 & \multirow[t]{2}{*}{160} & \\
\hline & 3.07 & 2.63 & 3.14 & 3.15 & 3.26 & & \\
\hline \multirow[t]{2}{*}{0.068} & & $\begin{array}{l}0.062 \\
0.153\end{array}$ & \multicolumn{3}{|c|}{ تأثير التداخلثر الثُاكثيز الكاينيتين } & \multicolumn{2}{|c|}{ LSD (0.05) } \\
\hline & \multicolumn{5}{|c|}{ متوسط تأثيرتداخل كلوريد الصوديوم × تركيز الكاينتين } & \multirow{2}{*}{\multicolumn{2}{|c|}{ تركيز كلوريد الصوديوم }} \\
\hline متوسط تأثيركلوريد الصوديوم & 100 & 75 & 50 & 25 & $\mathbf{0}$ & & \\
\hline 2.67 & 2.70 & 2.27 & 2.64 & 2.78 & 2.96 & & \\
\hline 3.23 & 3.20 & 2.73 & 3.55 & 3.22 & 3.46 & & \\
\hline 3.45 & 3.54 & 3.17 & 3.33 & 3.76 & 3.46 & & \\
\hline \multirow[t]{2}{*}{0.048} & \multicolumn{5}{|c|}{0.108} & \multicolumn{2}{|c|}{ LSD (0.05) } \\
\hline & \multicolumn{5}{|c|}{ متوسط تاثير تداخل مستوى السماد × تركيز الكاينيتين } & \multirow{2}{*}{\multicolumn{2}{|c|}{ مستوى السماد }} \\
\hline متوسط تأثير السماد & 100 & 75 & 50 & 25 & $\mathbf{0}$ & & \\
\hline 3.30 & 3.37 & 2.90 & 3.22 & 3.43 & 3.58 & \multicolumn{2}{|c|}{ صفر } \\
\hline 3.10 & 3.09 & 2.65 & 3.30 & 3.18 & 3.26 & \multirow{2}{*}{\multicolumn{2}{|c|}{$\begin{array}{c}160 \\
\text { LSD }(0.05)\end{array}$}} \\
\hline 0.039 & & & 0.08 & & & & \\
\hline
\end{tabular}


جدول (6): تاثير الرش بالكينتين واضافة سماد NPKZn في محتوى MDA ) Malondialdihyde ) (مايكرومول .غم وزن طري-1) لنبات الفلقل

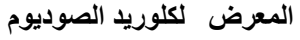

متوسط تاثير تاخل كلوريد

الصوديوم × مستوى السماد

1.93

1.75

3.00

2.74

3.97

3.76

0.046

متوسط تأثيركلوريد الصوديوم

1.84

2.87

3.86

0.033

متوسط تأثير السماد

2.97

2.75

$\mathbf{0 . 0 2 7}$

\begin{tabular}{|c|c|c|c|c|}
\hline \multicolumn{5}{|c|}{ تراكيز الكاينيتين ( جزء في المليون) } \\
\hline 100 & 75 & 50 & 25 & 0 \\
\hline 1.87 & 1.73 & 1.81 & 1.90 & 2.32 \\
\hline 1.81 & 1.46 & 1.56 & 1.81 & 2.10 \\
\hline 2.90 & 2.70 & 2.88 & 3.05 & 3.48 \\
\hline 2.78 & 2.40 & 2.76 & 2.82 & 2.95 \\
\hline 3.95 & 3.76 & 3.88 & 4.07 & 4.19 \\
\hline 3.77 & 3.62 & 3.73 & 3.81 & 3.86 \\
\hline 2.85 & 2.61 & 2.77 & 2.91 & 3.15 \\
\hline \multicolumn{5}{|c|}{ متوسطتاثيرتراكيز الكاينيتين } \\
\hline & 0.104 & \multicolumn{3}{|c|}{ تأثير التداخل الثلاثي } \\
\hline
\end{tabular}

متوسط تأثيرتداخل كلوريد الصوديوم × تركيز الكاينتين تراكيز الكاينيتين

\begin{tabular}{ccccc}
\multicolumn{5}{c}{ تراكيز الكاينيتين } \\
\hline 100 & $\mathbf{7 5}$ & $\mathbf{5 0}$ & $\mathbf{2 5}$ & $\mathbf{0}$ \\
1.84 & 1.60 & 1.69 & 1.86 & $\mathbf{2 . 2 1}$ \\
2.84 & 2.55 & 2.82 & 2.94 & 3.22 \\
3.86 & 3.69 & 3.81 & 3.94 & 4.03 \\
& & 0.073 & &
\end{tabular}

تركيز كلوريد الصوديوم

0

50

100

LSD (0.05)

متوسط تاثير تداخل مستوى السماد × تركيز الكاينيتين

تر اكيز الكاينيتين

\begin{tabular}{|c|c|c|c|c|c|}
\hline \multicolumn{5}{|c|}{ لراتير الحايليبين } & \multirow{2}{*}{ مستوى السماد } \\
\hline 100 & 75 & $\mathbf{5 0}$ & 25 & 0 & \\
\hline 2.91 & 2.73 & 2.86 & 3.01 & 3.33 & صفر \\
\hline 2.79 & 2.49 & $\begin{array}{c}2.68 \\
0.060\end{array}$ & 2.81 & 2.97 & $\begin{array}{c}160 \\
\text { LSD }(0.05)\end{array}$ \\
\hline
\end{tabular}

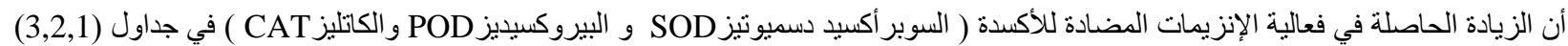

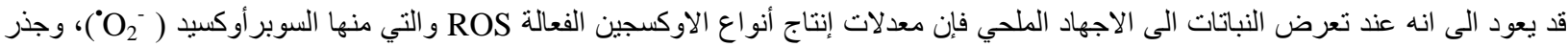

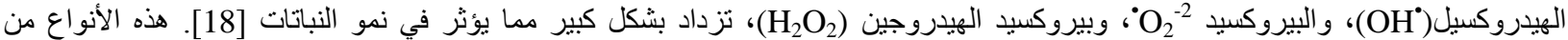

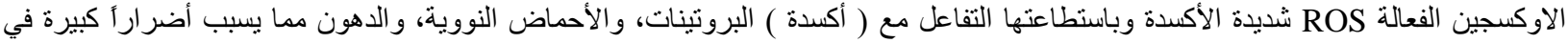

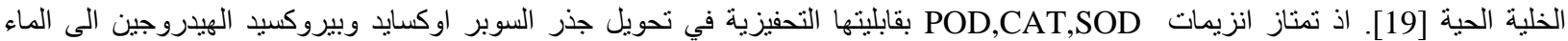

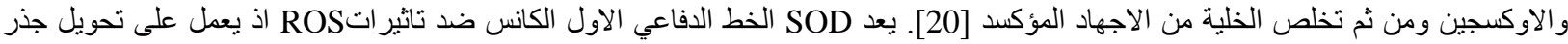

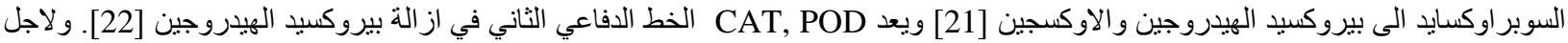

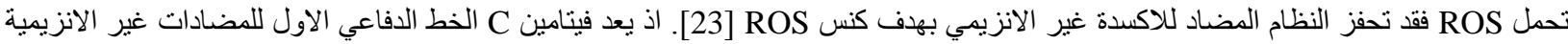

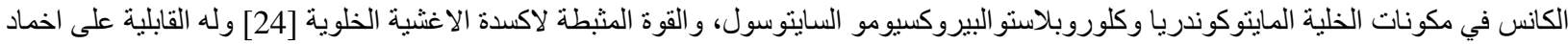

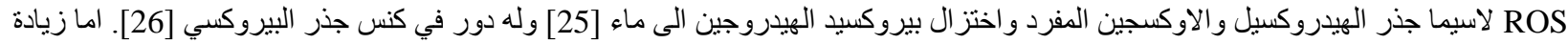

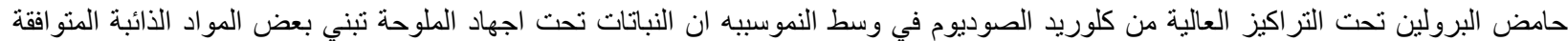

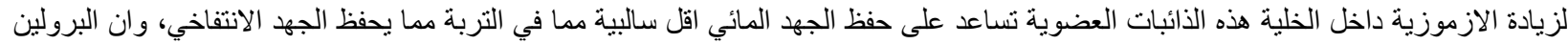

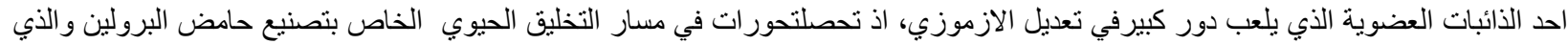

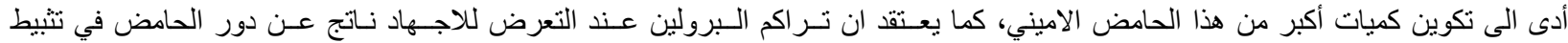

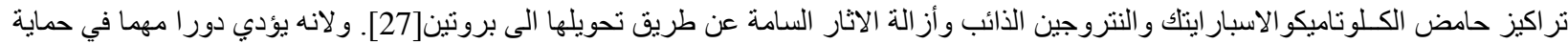

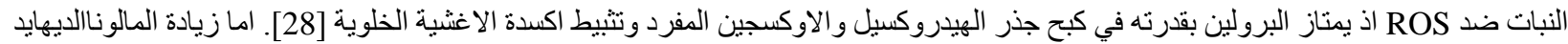

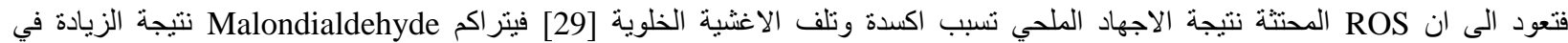
بيروكسيدات الاغشية اللبيدية واكسدة الاحماض الدهنية للاغشية الخلوية مما يتسبب في تهدم الاغشية الخلوية واختز ال في ثباتيتها [30].

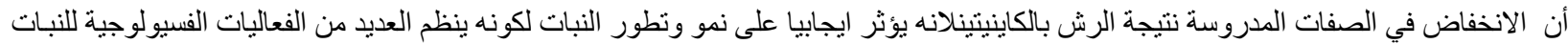

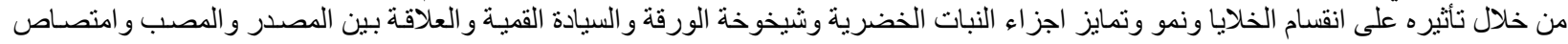

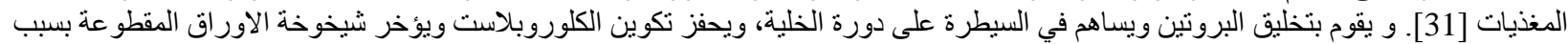

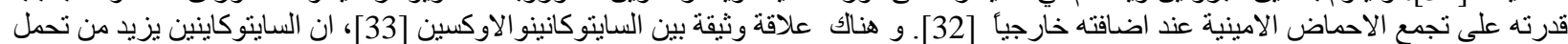

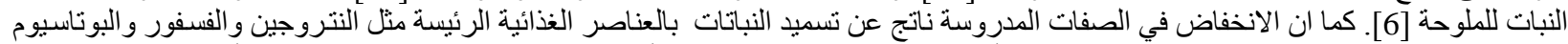

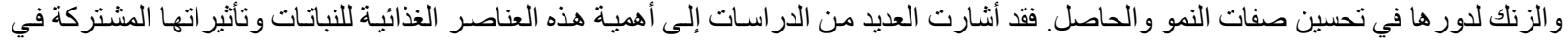

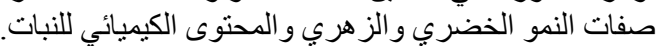




$$
\text { 1. زيدان السيد عبد العهال، عبد العزيز خلف الله، محمد الثال و محمد عبد القادر. (1977). (الخضر) الجزء الثاني (الإنتاج) دار المطبوعات الجديدة. }
$$

2.Navarro, M.J., Flores, P., Garrido, C. and Martinez V. (2006). Changes in the contents of antioxidant compounds in pepper fruits at different ripening stages, as affected by salinity. J.M. Navarro et al./ Food Chemistry. 96: 66-73

3.McCollum, J.P. (1980). Producing Vegetable Crop $3^{\text {rd }}$ ed. The Inter State Printer and Publisher. U.S.A.. P. 607.

4. Türkan, I. and Demiral, T. (2009). Recent developments in understanding salinity tolerance . Environmental and Experimental Botany. 67: 2-9.

5. Jaspers, P. and Kangasjärvi, J. (2010). Reactive oxygen species in abiotic stress signaling. Physiology Plantarum. 138(4):405-13.

6.Tran, L.S.P., Shinozaki, K. and Shinozaki, K.Y. (2010). Role of cytokinin responsive two-component system in ABA and osmotic stress signalings. Plant Signaling and Behavior. 5:2, 148-150.

7. Javid, G. M., Sorooshzadeh, A., Moradi, F., Mohammad, S. A., Sanavy, M. and Allahdadi, I. (2011). The role of phytohormones in alleviating salt stress in crop plants. Australian J. Crop Science. 5(6):726-734.

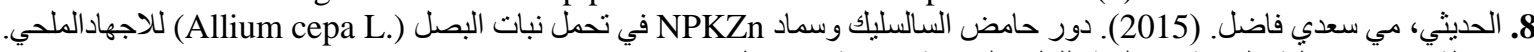

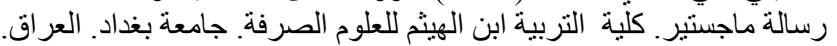

9. Pitotti, A., B.E., Elizalde and Anese, M. (1995). Effect of caramellzation and maillard reaction products on peroxidase activity. J. Food Biochem.18:445-457.

10.Beyer, W.F. and Fridovich, I. (1987). Assaying for superoxide dismutase activity: some large consequences of minor changes in conditions. Anal. Bio. chem. 161:559-566.

11. Nezih, M. (1985).The peroxidase enzyme activity of some vegetables and its resistance to heat. Food Agric. $36,877-880$

12.Aebi H. (1984). Catalase in Vitro Methods Enzymol. 105: 121-126.

13.Bradford, M. M. (1976). A rapid and sensitive method for the quantization of micro gram quantities of protein utilizing the principle of protein-dye binding analytical biochemistry. 72: $248-254$.

14.Hussain, I., Khan, L., Khan, M.A., Khan, F.U., Khan, S., and Khan, F.U . (2010). UV Spectrophotometeric Analysis Profile of Ascorbic Acid in Medicinal Plants of Pakistan. World Appl.Sci. J. 9(7):800-803.

15.Bates, L. S., R. P. Waldren and I. D. Teare. (1973). Rapid determination of free proline for water stress studies. Plant Sci. 39: 205-207.

16. Carmak, I. and J.H. Horst. (1991). Effects of aluminum on lipid peroxidation, superoxide dismutase, catalase, and peroxidase activities in root tips of soybean (Glycine max). Phsiol. Plant .83:463-468.

17. Little, T. M. and Hills, F. J. (1978). Agricultural Experimentation Design and Analysis. John Wiley and Sons, New York.

18. Kusvuran, S. (2010). Influence of drought stress on growth, ion accumulation and antioxidative enzymes in okra genotypes. Int. J. Agric. Biol. Vol. 14, No.3:401-406

19. Kafkas, E., Atasay, E. A., Sabir, F.K., Akgul, H. and Uckun, K. (2009). Effects of different irrigation intervals and fertilizer applications on certain chemical contents of Breabun applecultivar. African J. Biotechnol. 8:2138-2142.

20. Manivannan, P., Jaleel, C.A., Kishorekumar, A., Sankar, B., Somasundaram, R., Sridharan, R. and Panneerselvam, R. (2007). Changes in antioxidant metabolism of Vignaunguiculata L.walp., by propiconazole under water deficit stress. Colloids Surf. B. Biointerfaces. 57:69-74.

21. Zhang, X., Ervin, E., Evanylo, G., Sherony, C., Peot, C. (2005). Biosolids impact on tall fescue drought resistance J. of Residuals Sci. and Tech. 2:173-180.

22.Chgh, V., Kaur, N. and Gupta, A.K. (2011). Evaluation of oxidative stress tolerance in maize (Zea mays L.) seedlings in response to drought. Indian J.of Biochem. and Biophy. 48:47-53.

23.Alscher, R.A., Erturk, N. and Heath, L.S. (2002). Role of superoxide dismutases (SoDs) in controlling oxidative stress in plant. J. Exp. Bot. 53:1331-1341.

24.Quan, L.J., Zhang, B., Shi, W.W. and Li, H.Y. (2008 ). Hydrogen Peroxide in Plants, AVersatile Molecule of Reactive Oxygen Species Network. Supported by the National Natural Science Foundation of China, (30170238, 30670070).

25.Noctor,G.,Foyer, C.H. (1998). Ascorbate and glutathione: keeping active oxygen under control. Annu. Rev. Plant Physiol. Plant Mol Biol. 49, 249-279.

26.Sies, H. (1993). Strategies of antioxidant defence, Eur. J. Biochem. 215, 213-219.

27.Babu, M.A., Sing, D. and Gothandam, K. M. (2012). The effect of salinity on growth, hormones and mineral elements in leaf and fruit of tomato cultivar PKMI. J. Animal and Plant Sci. 22 (1) : 159-164.

28.Trovato, M., Mattioli, R. and Costantino, P. (2008). Multiple role of proline in plant stress tolerance and development, Redicontilincei. 19.325-346.

29.Cai, F., Mei, L.J., An, X.L., Gao, S., Tang, L., Chen, F. (2011). Lipid peroxidation and antioxidant responses during seed germination of Jatropha curcas. Int. J. Agric. Biol. 13(1): 25-30.

30.Dacosta, M., Huang, B. (2007). Changes in antioidant enzyme activities and lipid peroxidation for bent grass species in responses to drought stress. J. Am. Soc. Hortic. Sci. 132, 319-326. 
31.kieber, J. J. and Schaller, G.E. (2014). Cytokinins. The Arabidopsis Book 1l: eO168.doi:10.1199/tab.0168.

32.George, E.F., Hall, M.A. and Klerk, G.J.D. (2008). Plant Propagation By Tissue Culture. 3rd Edition, Springer. 205-226.

33.Jones, B., Gunnera, S.A., Petersson S.V., Tarkowski, P., and Graham, N. (2010). Cytokinin regulation of auxin synthesis in Arabidopsis involves a homeostatic feedback loopregulated via auxin and cytokinin signal transduction. The Plant Cell. 22: 2956-2969. 